\title{
A psychosocial rehabilitation approach for a case of persistent urogenital sinus with ambiguous genitalia in a female adult
}

\author{
Seema Chowdhary ${ }^{1}$, Arvind Kumar Chowdhary ${ }^{2}$, Sushma Pandey ${ }^{3}$ \\ ${ }^{1}$ Department of Obstetrics \& Gynaecology, Vydehi Institute of Medical Science \& Research Centre, Bangalore, India, ${ }^{2}$ Department of \\ Paediatrics, Vydehi Institute of Medical Science \& Research Centre, Bangalore, India, ${ }^{3}$ Department of Obstetrics \& Gynaecology, Patna \\ Medical College, Patna, India
}

\section{A B S T R A C T}

Objective: Urogenital Sinus is a rare congenital deformity which overture to preclude of normal embryonic vaginal progression. We report a case of urogenital sinus with ambiguous genitalia in a female adult. Case Report: An unmarried young female aged 26 years presented to our hospital with amenorrhea of 6 months and history of urinary incontinence from childhood. The diagnostic features of this case include normal haemogram with mildly elevated blood urea and creatinine, normal 17 ketosteroids, serum testosterone and DHEA; bilateral moderate hydronephrosis with uterus of size $6.8 \times 4.6 \times 5.3 \mathrm{~cm}$; urogenital sinus with partial separation of bladder and vagina-vaginal type; bilateral hydrouretronephrosis with mild renal cortical scarring and bicornuate unicollis uterus with cervix opening into the urogenital sinus. She was diagnosed with persistent urogenital sinus (Vaginal type) without congenital adrenal hyperplasia, but with associated mullerian anomaly. Treatment option was a U-shaped vaginoplasty with an aim of widening vaginal introitus and advancement of hypospadiac external urethral meatus to prevent micturition into vagina. Patient refused any surgery, and so, psychosocial treatment in form of personal hygiene and counselling was given to her. Conclusion: Though the possible treatment options were discussed, this case study emphasizes the need of psycho social counselling for the proper treatment of ambiguous genitalia.

Keywords: Persistent urogenital sinus, Ambiguous genitalia, surgical management, Psychiatric evaluation

\section{INTRODUCTION}

Disorders of the external genitalia are the major complication that burden parents, because of the unconscious emotional consequence of these reproductive structures and probably, the consequent impact of deformities on future generations. Persistent urogenital sinus is an extremely sporadic and variable urogenital deformity ${ }^{1,2}$ which may be due to the absence of urethrovaginal septum at 6 weeks gestation, overture to form single-exit chamber for the bladder and vagina to drain through. Large urogenital sinus (high confluence) which occurs at early stage of gestation, displays short vagina and a high urethral opening, whilst short urogenital sinus (low confluence) which forms at later stage of fetal development encompasses normal vaginal vestibule and low urethral opening. ${ }^{3}$ Prevalence of urogenital sinus has been reported as 0.6 in 10000 female births. ${ }^{4}$ From other congenital anomalies, urogenital sinus may differ in terms of involvement of organs, severity and complexity. The anomaly may be a simple anatomical defect, as a part of endocrinopathies or genetic aberrations. Each of these cases should be assessed precisely and treatment designed individually. In this communication, we represent a case of persistent urogenital sinus with ambiguous genitalia in an adult female. 


\section{CASE REPORT}

A 26-year-old young unmarried female was presented with the complain of 6 months' amenorrhea to our hospital for evaluation. Primarily she had medical history of urinary incontinence from childhood and tingling and numbness of the lower limbs since last 10 years. Bilateral ureteroureterostomy procedure was initially carried out at the age of 2 years and previous operation reports were not furnished. After some days the external wound was closed spontaneously. She had past evidence of irregular menstruations since begun of menarche at the age of 17 years and passage of urine and menstruation via same genital orifice. There was no record of consumption of any drugs or steroids by the mother of the patient during the antenatal period.

On general physical examination (GPE), the female was averagely built and nourished with a BMI of $20 \mathrm{~kg} / \mathrm{m}^{2}$. The facial appearance of the female was normal without any uncharacteristic pigmentation of skin. Vital signs of basic physiological functions are stable. Breast development of Tanner stage III and adult type of axillary hair were present. Pectus excavatum and a tuft of hair between L4-L5 with dimpling above gluteal cleft were evident. Anterior view of bilateral ureteroureterostomy shows a lower transverse abdominal scar. Genital examination revealed pubic hair of Tanner stage $\mathrm{V}$ (Figure 1). Labia minora was absent and a common urinary and vaginal orifice (measuring $0.5 \mathrm{~cm}$ ) was present that admitting tip of the little finger. Clitoris was slightly elongated (measuring $1.5 \mathrm{~cm}$ ) with prominent prepuce. Anus was in normal position and rectal investigation shown a small uterus (Figure 2).

Evaluation of blood test performed shows normal haemogram with mildly elevated blood urea and creatinine; normal 17 ketosteroids; serum testosterone and DHEA. Ultrasonography delineates bilateral moderate hydronephrosis with uterus of size $6.8 \times 4.6 \times 5.3 \mathrm{~cm}$. CT scan showed a persistent urogenital sinus, with partial separation of bladder and vagina-vaginal type; bilateral hydrouretronephrosis with mild renal cortical scarring; and bicornuate unicollis uterus with cervix opening into the urogenital sinus. Karyotype of the female was 46 XX. Cystoscopy and colposcopy showed a common narrow distal tunnel with the proximal wider vaginal canal with cervix directed posteriorly. Urethral meatus was situated $2 \mathrm{~cm}$ proximal to the external orifice.

A diagnosis of persistent urogenital sinus (Vaginal type) without congenital adrenal hyperplasia, but associated with mullerian anomaly was made. This was according to PVE classification. Treatment option suggested was a U-shaped vaginoplasty with an aim of widening vaginal introitus and

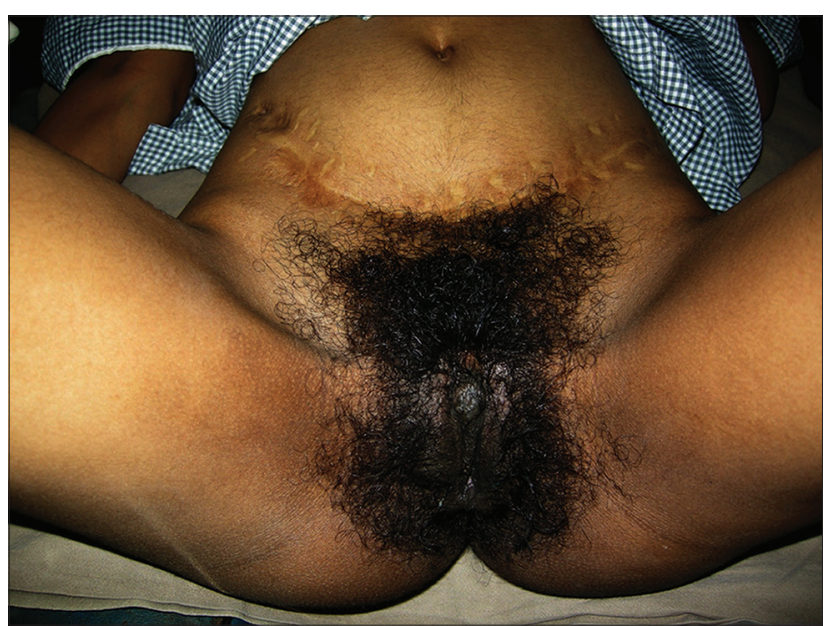

Figure 1:Evidence of abdominal scar, Tanner $\mathrm{V}$ Pubic hair and elongated clitoris

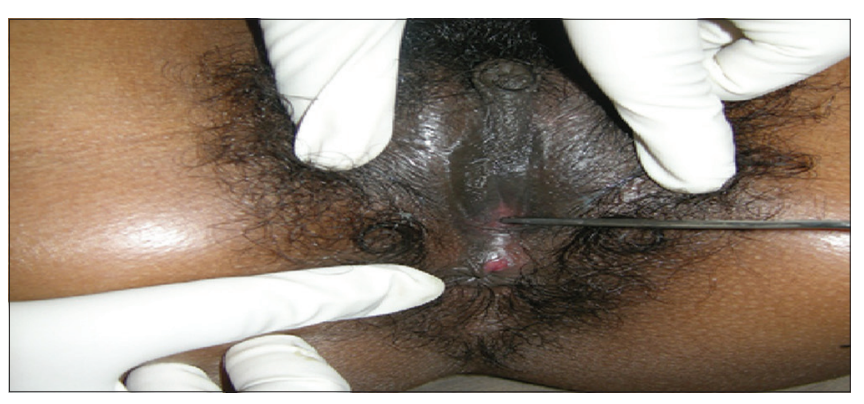

Figure 2:Common urinary and vaginal opening ,with anus in normal position

advancement of hypospadiac external urethral meatus to prevent micturition into vagina. Second option offered to the female was to create a urinary diversion.

Psychiatric evaluation was done to study the psycho social management of ambiguous genitalia. The patient was well educated female with good personal hygiene. She had no plans of marriage and found to be well adjusted in her environment with good family support. She has decided not to undergo any further surgical interventions. Finally, she was discharged after counseling for regular follow-up to conserve her renal function.

\section{DISCUSSION}

Persistent urogenital sinus deformity is a congenital disorder which provokes due to the retardation of normal embryonic cloacal progression during the early stage of fetal maturation. The molecular mechanism of this malformation is still obscure but from the results of different animal studies, the absence of Sonic hedgehog $(\mathrm{SHH})$ an endoderm derived signal molecule which induces mesodermal gene expression to play a crucial role in the development of urogenital sinus. ${ }^{5}$ Further many researchers 
reported an array of syndromes and anomalies associated with persistent urogenital sinus. ${ }^{6,7}$

Ambiguous genitalia and urogenital sinus anomalies represent a constellation of abnormalities with variable anatomy which encompasses clitoral hypertrophy, fusion of the labia with absence of the labia minor and common urogenital sinus. Similar observations were reported in our present case study. Management of children born with genital ambiguity and urogenital sinus anomalies is difficult and it is a potential task for urologist. Surgical interventions for the management of urogenital sinus include vaginal pull-through procedure, perineal flap vaginoplasty or a total urogenital mobilization. Further, rearrangement clitoral hypertrophy was highly warranted but restoration of clitoral innervation for optimal sexual gratification will be a positive outcome of the surgery. ${ }^{8}$

Psychological adjustment and self-appraisal after these surgeries are other significant squeal that have been insufficiently studied. These difficulties often evolve, irrespective of the surgical repair, as children grow to adulthood.

Focussed health education and reeducation of primary and secondary care physicians and healthcare workers on early diagnosis, initial interventions and need for early referral through regular workshop and public awareness programs; and finally local government may be compelled to increase healthcare funding to improve outcome and minimize psychosocial morbidity.

\section{REFERENCES}

1. Arena F, Romeo C, Cruccetti A, Antonuccio P, Basile M, Romeo $G$, et al. The neonatal management and surgical correction of urinary hydrometrocolpos caused by a persistent urogenital sinus. BJU Int 1999; 84:1063-1068.

2. Gul A, Yildirim G, Gedikbasi A, Gungorduk $K$ and Ceylan Y. Prenatal ultrasonographic features of persistent urogenital sinus with hydrometrocolpos and ascites. Arch Gynecol Obstet 2008; 278:493-496.

3. Hendren WH and Crawford JD. Adrenogenital syndrome. The anatomy of the anomaly and its repair. J Pediatr Surg 1969; 4:49-58.

4. Clavelli AA, H Watman E and Otaño, L. Persistent urogenital sinus. The Fetus [serial online] 2004. Available: http://www. thefetus.net/page.php?id=1275 via the internet.

5. Mo R, Kim JH, Zhang J, Chiang C, Hui CC, Kim PC, et al. Anorectal malformations caused by defects in sonic hedgehog signaling. Am J Pathol 2001; 159(2):765-774.

6. Arena F, Cordaro S, Romeo C, Scalfari G, Gitto L, Barbalace M, et al. Kaufman syndrome: Diagnostic and therapeutic problems. Pediatr Med Chir 1999; 21:67-71.

7. Stoler JM, Herrin JT and Holmes LB. Genital abnormalities in females with Bardet-Biedl syndrome. Am J Med Genet 1995; 55:276-278.

8. Diamond M and Sigmundson HK. Management of intersexuality. Guidelines for dealing with persons with ambiguous genitalia. Arch Pediatrics Adolescent Med 1997; 10:1046-1050. 